\title{
Why and how to spare the hippocampus during brain radiotherapy: the developing role of hippocampal avoidance in cranial radiotherapy
}

\author{
Tomas Kazda ${ }^{1,2^{*}}$, Radim Jancalek ${ }^{2,3}$, Petr Pospisil ${ }^{1}$, Ondrej Sevela ${ }^{4}$, Tomas Prochazka ${ }^{4}$, Miroslav Vrzal ${ }^{4}$, Petr Burkon ${ }^{1}$,
} Marek Slavik', Ludmila Hynkova', Pavel Slampa ${ }^{1}$ and Nadia N Laack ${ }^{5}$

\begin{abstract}
The goal of this review is to summarize the rationale for and feasibility of hippocampal sparing techniques during brain irradiation. Radiotherapy is the most effective non-surgical treatment of brain tumors and with the improvement in overall survival for these patients over the last few decades, there is an effort to minimize potential adverse effects leading to possible worsening in quality of life, especially worsening of neurocognitive function. The hippocampus and associated limbic system have long been known to be important in memory formation and pre-clinical models show loss of hippocampal stem cells with radiation as well as changes in architecture and function of mature neurons. Cognitive outcomes in clinical studies are beginning to provide evidence of cognitive effects associated with hippocampal dose and the cognitive benefits of hippocampal sparing. Numerous feasibility planning studies support the feasibility of using modern radiotherapy systems for hippocampal sparing during brain irradiation. Although results of the ongoing phase II and phase III studies are needed to confirm the benefit of hippocampal sparing brain radiotherapy on neurocognitive function, it is now technically and dosimetrically feasible to create hippocampal sparing treatment plans with appropriate irradiation of target volumes. The purpose of this review is to provide a brief overview of studies that provide a rationale for hippocampal avoidance and provide summary of published feasibility studies in order to help clinicians prepare for clinical usage of these complex and challenging techniques.
\end{abstract}

Keywords: Hippocampus, Hippocampal sparing, Hippocampal avoiding radiotherapy, Brain radiotherapy, Feasibility study, Planning study

\section{Background}

Both primary and secondary brain tumors (BT) represent a significant public health problem. An increasing incidence in primary brain tumors (PBT) as well as brain metastasis (BM) has been documented over recent years. In 2014, more than 24,000 new PBT are estimated to be diagnosed in the United States [1]. Moreover, about 1,4 million new solid tumor cases of all histological origin are diagnosed each year in the United States and approximately 30\% of

\footnotetext{
* Correspondence: tomas.kazda@mou.cz

'Department of Radiation Oncology, Faculty of Medicine, Masaryk University and Masaryk Memorial Cancer Institute, Zluty kopec 7, 65653 Brno, Czech Republic

${ }^{2}$ International Clinical Research Center, St. Anne's University Hospital Brno, Pekarska 53, 65691 Brno, Czech Republic

Full list of author information is available at the end of the article
}

them develop BM [1]. Therefore, management of BT is an increasingly important component of cancer therapy [2].

Radiotherapy is an important modality in the treatment of BT. Radiotherapy remains the standard treatment for vast majority of high-grade or malignant brain tumors and plays an integral role in treatment of many low-grade and benign primary brain tumors. However, concerns regarding neurocognitive toxicity after radiotherapy in patients with benign or low-grade tumors make the timing of treatment controversial [3].

Historically, radiotherapy was also a mainstay of treatment for BM. With improved survival and increased awareness of the cognitive effects of WBRT, the role of WBRT in BM has come under question [4]. Because of these concerns there has been a trend towards increased reliance on focal treatments such surgery and stereotactic 
radiosurgery (SRS) [5]. However, achieving whole brain control is associated with improved survival and preserved neurocognitive domains with exception of memory function, especially recall and delayed recall [6]. Thus, understanding the risk of brain tumor recurrence at distant sites of brain is important in counseling patients regarding the risks and benefits of WBRT. Patients with single BM and no extracranial metastases are at low risk for in-brain recurrence and omitting early WBRT because of the risk of intermediate and late adverse effects (AE) can be safely done as long as the patient commits to regular imaging [7]. Conversely, patients with progressive systemic disease are at a higher risk for distant brain failure and likely benefit from the addition of WBRT despite possible late complications [8].

For most malignant adult $\mathrm{PBT}$ and BM, radiotherapy prolongs survival but is rarely curative. Thus, emphasis on minimizing the $\mathrm{AE}$ of treatment is becoming one of the most important factors in the treatment. Recently, more attention has been paid to symptom related outcomes of care, especially to neurocognitive function (NCF) and quality of life (QoL) [9-11]. With improvements in radiotherapy systems technology, it is now possible to modify treatment plans to selectively spare structures that may contribute to decreased QoL and NCF. In order to achieve this aim, it is important to determine appropriate end-points primarily in relation to the ongoing randomized clinical trials as resources for future treatment guidelines [12].

Decline in NCF as an iatrogenic side effect of brain irradiation is well-known [13]. The mechanism of radiation injury is complex and multi-factorial. In the past, cognitive decline after radiotherapy was believed to be a late effect of treatment mediated through microvascular changes and neuroglial loss. However, there is increasing evidence for acute and subacute cognitive changes after radiotherapy that appear to be mediated through the neurogenic zones including the hippocampus. Preclinical evidence supports the concept of hippocampal radiation injury as a mediator of subsequent $\mathrm{AE}$, most notably in memory-related domains of neurocognition [14]. Retrospective clinical reports as well as early results of prospective trials support the role of hippocampus in early changes in cognitive function after radiotherapy [11]. The purpose of this review is to provide a brief overview of studies that provide a rationale for hippocampal avoidance (HA) and provide summary of published feasibility studies in order to help clinicians prepare for clinical usage of these complex and challenging techniques.

\section{Hippocampus and radiation injury}

The hippocampus is a paired brain structure, located in the ventromedial part of the temporal lobes, laying lateral to the temporal horn of the lateral ventricle. The hippocampus is composed of the dentate gyrus and the cornu ammonis regions and belongs to the limbic system. Its main role in brain function is cooperation in learning, consolidation and retrieval of information and it is also essential for formation of new memories [15]. Bilateral and unilateral radiation injury of the hippocampus is known to alter learning and memory formation [16]. Complete pathophysiologic explanation of all these processes is still lacking; nevertheless, the role of neurogenesis seems to be one of the most compelling [17].

Mitotically active neural stem cells (NSCs) are located in different parts of brain, namely in the subependymal zone and in the subgranular zone of the dentate gyrus, wherefrom they migrate into the granular cell layer of hippocampus [18]. The hippocampal subgranular zone is a critical neurologic center for learning and memory [19]. NSCs have typical features of the stem cells. They are capable of both self-renewal and generating new differentiated cells [20]. Neurogenesis is a complicated process with integration of many regulatory cells as astrocytes or endothelial cells with coordinate evolution of neural precursor cells together with each other in a specific neurogenic microenvironment called "niche" [21,22].

Multiple preclinical studies support the hypothesis of hippocampus-mediated cognitive dysfunction [23-30]. In vivo animal studies demonstrate sensitivity of these NSCs to ionizing radiation. Apoptosis of NSCs after ionizing radiation was first described in the subependymal zone in the young adult rat. After single x-ray doses of 5 or $30 \mathrm{~Gy}$, apoptosis peaked 6 hours after irradiation [23]. Several years later, postradiation apoptosis was observed also in the rats' dentate gyrus after exposure to single 10 Gy dose [24]. Decline in neurogenesis was associated with cognitive impairment in rodent models for both single and fractionated brain irradiation [14,25]. Mizumatsu et al. irradiated the whole brain of experimental mice with various single doses and used immunohistochemical staining methods for detection of apoptosis as well as numbers of proliferating cells and immature neurons in the subgranular zone of the hippocampal dentate gyrus. Dose-dependent apoptosis was observed and peaked 12 hours after irradiation followed by subsequent reduction in amount of proliferating cells in subgranular zone [27]. Changes in neurogenesis were associated with an inflammatory response as validated by detection of activated microglia cells [29]. Moreover, administration of anti-inflammatory agents such as ramipril and indomethacin can mitigate radiation-induced cognitive impairment in rodents suggesting the inflammatory response is important in mediating the effects of radiotherapy [30].

However, these and other mechanisms of radiation effects on neurogenesis do not completely describe the radiobiology of the hippocampus [31]. More recent in vitro and in vivo research reveals other important radiation induced 
hippocampal changes which may also influence cognition [32]. Investigators at University of California at Irvine, CA, USA have optimized a SYBR green based assay to study the effects of low dose RT before changes are visible radiographically. Even a dose of 2 Gy delivered to human NSCs leads to decreased numbers of cells undergoing neuronal differentiation after irradiation [33]. Additional work from the same group suggests the mechanism of radiation-induced inhibition of neurogenesis may be mediated through oxidative stress [34].

Although significant pre-clinical data supports a decrease in NSC number and function after radiotherapy, changes in neuronal architecture, as recently described by Parihar and Limoli in measurements of micromorphometric parameters in mice following cranial irradiation by 1 and 10 Gy, may also be important in mediating the effects of radiotherapy [35]. Dose-dependent reduction of dendritic branching, length and area were described as well as the reduction of immature filopodia as compared with mature spine morphology of dendritic segments. These postradiation changes correlated with alterations in synaptic protein production that were noted up to 1 month after brain irradiation [35]. These types of changes are likely to be equally important as disruption of neurogenesis in eliciting cognitive decline after radiotherapy.

Recent studies provide dose-response data and estimation of clonogenic survival fraction of human NSCs after brain irradiation. This data is important from a radiation oncology point of view primarily for determination of pertinent treatment planning recommended dose constraints.

In QUANTEC analysis, the normal brain $\alpha / \beta$ value has been established to be 2.9 [36]. For the hippocampal region, most authors use the $\alpha / \beta$ ratio in range from 2 to 3 [37]. However, other authors work with the $\alpha / \beta$ value for NSCs compartments equal to $10[38,39]$ using a general value established for stem cells [40]. Some authors use $\alpha / \beta$ ratio 10 for the true hippocampus and an $\alpha / \beta$ value 2 for the whole hippocampus planning-at-risk volume illustrating the lack of consensus regarding the optimal model of radiation sensitivity. However, preclinical evidence suggests doses as low as 2 Gy to result in apoptosis of neurogenic stem cells supporting a no-shoulder dose-response [29,33]. Several other studies have measured altered survival and proliferation using a range of metabolic and SYBR green based assays. These studies revealed that doses of as low as 2 Gy reduced survival by over 50\% [33]. Thus, accumulating preclinical data indicate that neurocognitive dysfunction manifests at much lower doses (<10 Gy) than previously expected [34].

\section{Clinical evidence for hippocampal sparing}

In addition to preclinical evidence, retrospective clinical reports also suggest the hippocampal region may play a role in NCF decline after radiotherapy. Children with brain tumors treated on prospective clinical trials that included planned neurocognitive assessments were evaluated with neurocognitive studies up to 5 years after radiotherapy. Mean doses of $45 \mathrm{~Gy}$ or higher to the left temporal lobes were associated with significant declines in longitudinal IQ $[41,42]$. The relationship between hippocampal dose level and the risk of subsequent NCF impairment was described in the group of patients with adult low-grade gliomas; NCF was assessed at the baseline and at 18 months follow-up for conventionally treated patients. Biologically equivalent dose greater than 7.3 Gy (equivalent dose in 2-Gy fractions) applied to $40 \%$ of hippocampal volume was associated with long-term NCF impairment, especially in list-learning delayed recall [43]. Recently, results of the first prospective phase II study (RTOG 0933) of $\mathrm{HA}$ in BM patients suggest a reduction in risk of cognitive dysfunction with HA [11]. Primary cognitive outcome was delayed recall at 4 months as measured by the Hopkins Verbal Learning Test for patients with WBRT comparing with those with HA WBRT. Results were compared to historical control group. Only $7 \%$ of patients experienced decline in memory compared to $30 \%$ of patients in the historical cohort $(\mathrm{p}=0.0003)$. QoL was evaluated as well and was preserved up to 6 months follow-up. Based on these results, RTOG is planning a phase III randomized trial of prophylactic cranial irradiation with or without hippocampal sparing for small cell lung cancer patients (RTOG 1317).

\section{Hippocampus-sparing: feasibility studies}

Although evidence is mounting in regards to the importance of the hippocampus in mediating cognitive changes after radiotherapy, it has only been with recent technologic advances that the feasibility of a meaningful reduction in hippocampal dose while maintaining acceptable tumor control probability has been established. Below we review the feasibility studies evaluating $\mathrm{HA}$ for PBT and BM (Figure 1).

\section{Primary brain tumors: HA feasibility studies}

Complex intensity modulated radiotherapy (IMRT) plans were developed to evaluate the feasibility of sparing contralateral and bilateral hippocampi in glioma patients [38]. In "sparing" plans for the hemispheric HGG cases, it was possible to reduce the mean physical dose to the contralateral hippocampus planning-at-risk volume by $56.8 \%$ compared to standard treatment plan prepared without prospective sparing of the hippocampus (15.8 Gy vs. 36.6 Gy). In addition, more central location of PBT enables sparing of both hippocampi as documented by mean physical dose reduction by more than a third (16.8 Gy in sparing vs. $25.6 \mathrm{~Gy}$ in standard plan) [38]. Based on previous preclinical data, even low doses can result in NSCs apoptosis, but assuming that hippocampus is 


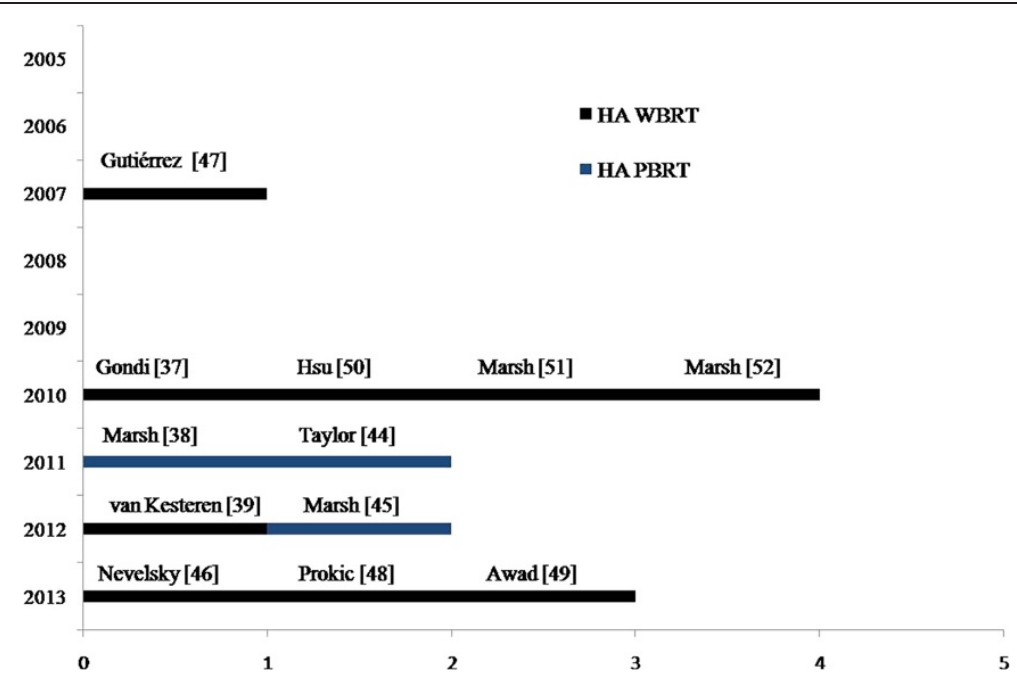

Figure 1 Overview of recently published radiotherapy planning studies dealing with hippocampal avoidance. Only studies with more than 9 patients included [44].

more parallel than serial organ, a reduction of median hippocampal dose may reduce NCF impairment even if no unequivocal cut-off dose threshold is known. Unfortunately, reported dose reduction is related to hippocampal planning-at-risk volumes, which were created by $3 \mathrm{~mm}$ expansion of hippocampi, so it is not possible to compare these results with other published studies.

PBT are frequent diagnosis in children in whom minimization of subsequent late $\mathrm{AE}$ is even more important. In a evaluating HA in pediatric gliomas, the NSCs compartments, the limbic circuit and the whole hippocampus were recognized as organ at risk (OARs) and included in experimental treatment plans [45]. For each RT plan the biological equivalent doses were calculated providing more radiobiologically exact comparison of doses in OARs. In all cases (10 different PBT), experimental plans significantly reduce both mean physical dose (by $56.0 \%$ ) and mean biological equivalent doses (by 52.1\%) delivered to the study OARs in comparison to plans without any effort to spare these structures. As might be expected, greatest hippocampal sparing was seen in hemispheric gliomas whereas worse results were observed for diffuse tumors where whole ventricular RT was indicated.

\section{Brain metastases: HA feasibility studies}

Many planning studies have shown the dosimetric feasibility of HA WBRT using different radiotherapy systems as linear accelerator (LINAC) based IMRT, helical tomotherapy or volumetric modulated arc therapy (VMAT). In addition, HA brain irradiation is also possible using Elekta IMRT step and shoot systems [46].

The pioneer study was performed in 2007 by Gutiérrez et al., who tested feasibility of HA WBRT with simultaneous integrated boost (SIB) to BM in experimental radiotherapy plans using helical tomotherapy [47]. Regardless a different setting of treatment plans (pitch and field width), they described no significant difference in hippocampal doses. Authors concluded that it is possible to create combined plans with homogeneous whole brain dose distribution equivalent to conventional WBRT, while conformal HA and radiosurgically equivalent dose boosting to individual metastases.

Mean dose guidelines for HA WBRT were first published by Gondi [37]. HA plans were compared with standard WBRT ones where a homogenous dose $30 \mathrm{~Gy}$ was applied to the whole brain including hippocampus. For HA plans, the median hippocampal dose was achieved 5.5 Gy ( $\left.D_{\max } 12.8 \mathrm{~Gy}\right)$ and 7.8 Gy ( $\left.\mathrm{D}_{\max } 15.3 \mathrm{~Gy}\right)$ for helical tomotherapy and LINAC based RT, respectively. These dose reductions have been considered a reference for other subsequent planning studies.

Because of higher availability of stereotactic systems, recent trends are to combine WBRT with stereotactic boosting to $\mathrm{BM}$ and thus improve local control. Also in this sequential concept, it is possible to spare hippocampus in both parts of treatment: HA WBRT and subsequent HA SRS boost. Moreover, using IMRT, it is possible to integrate boosting into the first WBRT part in concept of SIB. Comparing this approach with classical sequential concept (WBRT + stereotactic radiotherapy), the SIB is more effective in lowering doses to the hippocampus for patients with up to 8 metastases [48].

Although multiple techniques allow HA WBRT, treatment time can vary significantly depending on the technique. Using VMAT for HA WBRT with SIB for melanoma brain metastases, the average beam-on time was achieved 3.6 min while abide the RTOG 0933 feasibility DV constraints [49]. Arc based delivery times are generally faster 
than conventional IMRT. VMAT was 3.5 times faster than classical IMRT techniques as discussed in other one planning study focused on HA WBRT published last year $[46,50]$.

Similarly as for BM treatment, HA WBRT technique can be used for prophylactic cranial irradiation (PCI) where are NCF preserving approaches even more justified. Comparisons of limbic sparing experimental plans were conducted in 11 patients indicated for WBRT and for PCI. Similar reduction of hippocampal biological equivalent doses was achieved for both of these clinical situations $[51,52]$. These results are not surprising considering the fact, that PCI differ from WBRT only in terms of fractionation and that the standard radiotherapy technique is similar.

Comparison of treatment planning results with other studies is summarized in Table 1.

\section{How to spare hippocampus}

Because of hippocampal anatomic shape and central brain location, it can be a challenge to create appropriate HA treatment plan for irradiation of both PBT and BM. Nevertheless, modern IMRT techniques such as helical tomotherapy or VMAT are able to achieve HA with acceptable target volume coverage and dose homogeneity. Although a variety of treatment techniques are available for HA WBRT, the ability to achieve OAR dose goals varies by technique. In general, helical tomotherapy offered significantly better HA compared to LINAC based IMRT in terms of the mean normalized tissue dose, as well as the median and maximal hippocampal dose. However, despite different technical capabilities of mentioned radiotherapy systems, it can be concluded, that using either helical tomotherapy or LINAC based IMRT is sufficient for sparing not only traditional OARs but also the hippocampus [37]. An effort to minimize hippocampal dose must not lead to irradiation of other brain OARs. This is important especially for gliomas treated by overall higher doses compared with treatment of BM.

In addition, dose constraints are expected to be different for HA WBRT and HA PBRT. For BM, it is possible to sufficiently spare both hippocampi, and dose-volume constraints used in representative HA WBRT planning studies were summarized in Table 1 . On the other hand, for HA PBRT, especially in the treatment of hemispheric gliomas, ipsilateral hippocampus is often included in target volumes (and considering much larger doses compared with WBRT). Thus, it is not possible to achieve appropriate dose reduction for ipsilateral hippocampus and only contralateral hippocampus could be considered as OAR. As an example, for HGG the following constraints criteria have been proposed: $0 \%$ of the hippocampal volume cannot receive more than 8 Gy in the first phase of treatment (up to $46 \mathrm{~Gy}$ ) and no more than 4 Gy in the final phase (next 14 Gy to the target volume) [38].

Contouring of target volumes is, as a potential source for systematic error, one of the most important parts of whole radiotherapy planning process. Structure contouring for radiotherapy purpose is sometimes slightly different process comparing with the other medical discipline.

Table 1 Hippocampal dose-volume constraints and achieved doses in representative HA WBRT planning studies

\begin{tabular}{|c|c|c|c|c|c|c|c|c|c|c|c|}
\hline \multirow[t]{2}{*}{ Author, year } & \multirow[t]{2}{*}{$\begin{array}{l}\text { Clinical } \\
\text { situation }\end{array}$} & \multirow[t]{2}{*}{ RT system } & \multirow[t]{2}{*}{ No. } & \multirow[t]{2}{*}{ Fractionation } & \multicolumn{2}{|c|}{$\begin{array}{l}\text { Hippocampal } \\
\text { constraints }\end{array}$} & \multicolumn{3}{|c|}{ Hippocampal doses } & \multirow{2}{*}{\multicolumn{2}{|c|}{$a / \beta$}} \\
\hline & & & & & $D_{\max }$ & & $D_{\max }$ & $D_{\text {mean }}$ & $\mathrm{D}_{\text {median }}$ & & \\
\hline Gutierrez, 2007 [47] & WBRT & $\mathrm{HT}$ & 10 & $15 \times 2.15$ Gy & $6 \mathrm{~Gy}$ & & - & $5.86 \mathrm{~Gy}_{2}$ & $5.34 \mathrm{~Gy}_{2}$ & 2 & \\
\hline \multirow[t]{2}{*}{ Gondi, 2010 [37] } & WBRT & HT & 5 & $10 \times 3.0 \mathrm{~Gy}$ & $6 \mathrm{~Gy}$ & 3 Gy $\leq 20 \%$ & $12.8 \mathrm{~Gy}$ & - & $5.5 \mathrm{~Gy}$ & 2 & \\
\hline & & LINAC & & & 11 Gy & 9 Gy $\leq 40 \%$ & $15.3 \mathrm{~Gy}$ & & $7.8 \mathrm{~Gy}$ & 2 & \\
\hline Hsu, 2010 [50] & $W B R T+S I B$ & LINAC & 10 & $\begin{array}{l}15 \times 2.15 \text { Gy } \\
\text { (SIB á } 4.2 \text { Gy) }\end{array}$ & - & $\mathrm{D}_{\text {mean }}<6 \mathrm{~Gy}_{2}$ & - & $5.23 \mathrm{~Gy}_{2}$ & - & 2 & \\
\hline \multirow[t]{2}{*}{ Marsh, 2010 [51] } & $\mathrm{PCl}$ & HT & 11 & $15 \times 2.0 \mathrm{~Gy}$ & 15 Gy & & - & 12.5 Gy & - & - & \\
\hline & WBRT & & 11 & $14 \times 2.5 \mathrm{~Gy}$ & 15 Gy & & & 14.3 Gy & & & \\
\hline \multirow[t]{2}{*}{ Marsh, 2010 [52] } & $\mathrm{PCl}$ & HT & 10 & $15 \times 2.0 \mathrm{~Gy}$ & & & - & 11.5 Gy & - & - & \\
\hline & WBRT & & 10 & $14 \times 2.5$ Gy & - & - & - & 11.8 Gy & - & - & \\
\hline Van Kesteren, 2012 [39] & WBRT & LINAC 3D-CRT & 10 & $12 \times 2.5 \mathrm{~Gy}$ & & & $13.5 \mathrm{~Gy}$ & $6 G y$ & - & 10 & \\
\hline Nevelsky, 2013 [46] & WBRT & LINAC IMRT & 10 & $10 \times 3.0 \mathrm{~Gy}$ & 16 Gy & $\mathrm{D}_{100 \%}<9 \mathrm{~Gy}$ & 14.35 Gy & - & - & - & \\
\hline Awad, 2013 [49] & WBRT + SIB & VMAT RA & 30 & $5-15 f x$ & - & & $32.2 \mathrm{~Gy}$ & 20.4 Gy & $21.9 \mathrm{~Gy}$ & - & \\
\hline \multirow[t]{2}{*}{ Prokic, 2013 [48] } & $W B R T+S I B$ & VMAT RA & 10 & $\begin{array}{l}12 \times 2.5 \text { Gy } \\
\text { BM } 12 \times 4.25\end{array}$ & - & & 12.33 Gy $\left(D_{2 \%}\right)$ & 7.55 Gy & 7.15 Gy & $\mathrm{H} 2$ & BM 10 \\
\hline & WBRT + FSRT & VMAT RA & 10 & $\begin{array}{l}12 \times 2.5 \mathrm{~Gy}+ \\
\text { FSRT } 2 \times 9 \mathrm{~Gy}\end{array}$ & - & & $15.82 \mathrm{~Gy}\left(\mathrm{D}_{2 \%}\right)$ & $9.8 \mathrm{~Gy}$ & 9.34 Gy & $\mathrm{H} 2$ & BM 10 \\
\hline
\end{tabular}


Exact volumetric assessing of the whole hippocampus is important especially in basic neurological research [53] as well as in research dealing with diseases connected to hippocampal impairment [54]. On the other hand, only some HA radiotherapy feasibility planning studies defined in detail the process of contouring, almost exclusively with reference to the Radiation Therapy Oncology Group online contouring atlas [55]. Authors of this atlas do not contour the entire hippocampus, but are focusing mostly on the subgranular zone as a place of NSCs occurrence. This approach is suggested as a standard for HA WBRT. On the other hand, for HA PBRT, where only the contralateral hippocampus is often spared, it is possible to contour the whole hippocampus irrespective of its NSCs rich part according to a radiation oncologist's guide to contouring the hippocampus proposed by Chera et al. [56]. Moreover, considering an attempt to spare NCF during brain irradiation, some studies defined OARs even more comprehensively including the whole limbic circuit (whole hippocampus; the rest of limbic circuit comprising the amygdalar complex, the fornix, the cingulum, the cingulated gyrus, and the mammillary bodies) [38,51].

Physician's requirements expressed in terms of dosevolume constraints are best achievable using inverse planning, which enables to set different priority points to different OARs and target volumes and thus it is possible to find compromise in dose coverage for all important treatment structures. As an example, in Table 1 were described parameters for both helical tomotherapy and LINAC based IMRT as presented in a seminal planning study [37]. These set of dose-volume constraints have been used in RTOG 0933 study, a first HA WBRT clinical trial which results were mentioned above [11]. Although methodology of the study enables differences in radiotherapy systems for preparation of a particular treatment plan, plans have to meet the required dosimetric constraints prior to approval for clinical use.
Table 2 presents acceptable and unacceptable variations from "per protocol planning" for including particular IMRT treatment plan into RTOG 0933 trial. In our HA planning study, we compare different Arc radiotherapy techniques in order to achieve mentioned constraints. In the setting of non-coplanar beams arrangement, we have observed even higher hippocampal preservation compared to classical coplanar irradiation (not published data) (Figure 2).

In addition to the recent sophisticated methods enabling HA during brain irradiation, a simpler technique using two opposing laterolateral fields with central leaf shielding for appropriate HA has also been discussed [39]. The simplicity of this technique could enable radiotherapy departments that are not equipped with the latest technology to still offer HA radiotherapy. This technique is simple reproducible, as demonstrated by one experimental plan from our department (Figure 3).

\section{Controversies}

There are several medical and ethical controversies especially about the indications for HA brain irradiation. Providing of hippocampal sparing techniques is difficult and expensive. Thus, responsible decision must be made with respect to selection of appropriate patients especially in terms of probability of long term survival and QoL. The difference in the cost of basic 3D-CRT and advanced radiotherapeutic methods needed for HA brain irradiation is probably the most important controversy. Unfortunately, in many departments, especially in lowincome countries, IMRT techniques are not widely available even for curative treatment (head and neck or prostate cancer). And even in large centers, it is not clear, whether implementation of more expensive RT technique is worthwhile to prevent probable mild neurocognitive decline. Only well designed randomized trials and cost-effective analysis can evaluate whether, or not these approaches should be incorporated into general practice. On the other

Table 2 Acceptable and unacceptable variations from per protocol IMRT treatment planning according to RTOG 0933 trial [11]

\begin{tabular}{|c|c|c|c|c|}
\hline Treatment component & Parameter & Per protocol & Variation acceptable & Unacceptable deviation \\
\hline \multirow[t]{2}{*}{ MRI/CT Fusion and Contouring } & MRI-CT fusion & $\begin{array}{l}\text { No corrections to MRI/CT } \\
\text { fusion requested }\end{array}$ & $\begin{array}{l}\text { No corrections to MRI/CT } \\
\text { fusion requested }\end{array}$ & $\begin{array}{l}\text { Corrections to MRI/CT } \\
\text { fusion requested }\end{array}$ \\
\hline & Hippocampal Contouring & $\begin{array}{l}\leq 2 \mathrm{~mm} \text { deviation using } \\
\text { the Hausdorff distance* }\end{array}$ & $\begin{array}{l}>2 \text { and } \leq 7 \mathrm{~mm} \text { deviation } \\
\text { using the Hausdorff distance* }\end{array}$ & $\begin{array}{l}>7 \mathrm{~mm} \text { using the } \\
\text { Hausdorff distance* }\end{array}$ \\
\hline \multirow[t]{4}{*}{ HA WBRT IMRT Planning } & PTV & $D_{2 \%} \leq 37.5 \mathrm{~Gy}$ & $\mathrm{D}_{2 \%}>37.5 \mathrm{~Gy} \leq 40 \mathrm{~Gy}$ & $V_{30}<90 \%$ \\
\hline & & $D_{98 \%} \geq 25 \mathrm{~Gy}$ & $\mathrm{D}_{98 \%}<25 \mathrm{~Gy}$ & $\mathrm{D}_{2 \%}>40 \mathrm{~Gy}$ \\
\hline & Hippocampus & $\mathrm{D}_{100 \%} \leq 9 \mathrm{~Gy}$ & $\mathrm{D}_{100 \%} \leq 10 \mathrm{~Gy}$ & $\mathrm{D}_{100 \%}>10 \mathrm{~Gy}$ \\
\hline & & $D_{\max } \leq 16 \mathrm{~Gy}$ & $\mathrm{D}_{\max } \leq 17 \mathrm{~Gy}$ & $D_{\max }>17 \mathrm{~Gy}$ \\
\hline OARs constraints & Optic nerves and chiasm & $\mathrm{D}_{\max } \leq 37.5 \mathrm{~Gy}$ & $\mathrm{D}_{\max } \leq 37.5 \mathrm{~Gy}$ & $\mathrm{D}_{\max }>37.5 \mathrm{~Gy}$ \\
\hline Unscheduled break days & - & 0 break days & $1-3$ break days & $>3$ break days \\
\hline
\end{tabular}

*according to comparison with contours prepared by co-principal investigators.

PTV: planning target volume; $D_{2} \%$ : dose in $2 \%$ of volume; $D_{98 \%}$ : dose in $98 \%$ of volume; $D_{100 \%}$ : dose in $100 \%$ of volume; $D_{\text {max }}$ : maximal dose; $V_{30}$ : volume irradiated by 30 Gy. 


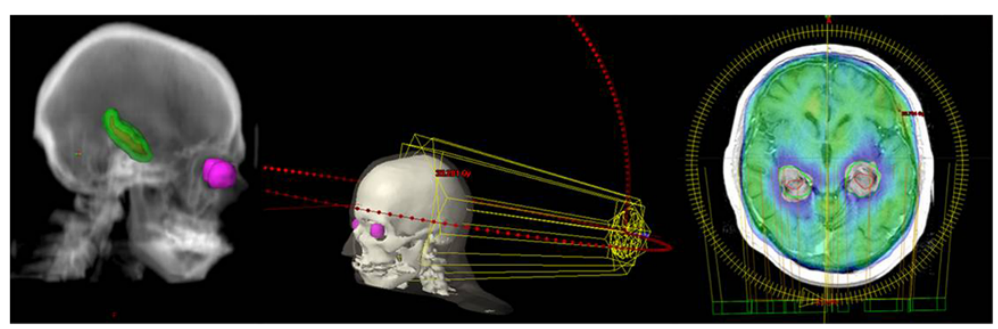

Figure 2 Examples of non-coplanar Arc treatment plan with hippocampal sparing and homogenous dose coverage in the rest of the brain.

hand, if IMRT is indicated for another reason, the hippocampus can be considered as other OAR assuming that no other standard OAR will receive more radiation.

Especially for patients with PCI or for children, it is important consider potential $\mathrm{AE}$ of some techniques using for hippocampal sparing such as helical tomotherapy for example. In an effort to minimize dose in hippocampus, there is a risk of overtreatment of other parts of the brain and surrounding structures with increased risk of induced secondary malignancies. To assess this potential disadvantage of helical tomotherapy, one study measured integral doses in uninvolved brain regions of HGG patients for both conventional IMRT and helical tomotherapy techniques [57]. The results proved reduction of brain integral dose in average by $23 \%$ after IMRT compared with tomotherapy in all tested treatment plans. Despite a theoretical risk of local overtreatment, integral dose delivered by any technique has been surprisingly lower in sparing plans compared with non-sparing ones. From this point of view, usage of traditional IMRT techniques is considered as optimal way how to spare hippocampus.
Another approach reducing brain integral dose would be the proton therapy [58].

Considering sparing of some part of brain during WBRT in BM treatment as well as in prophylactic situation, a worry of subsequent increase risk of intracranial disease progression in spared regions is justified. However, many imaging studies described overall low number of metastases in the hippocampus as well as in other parts of the limbic circuit [59-62]. For example, on study evaluated $697 \mathrm{BM}$ in 107 patients, only one of 53 oligometastatic patients $(1.9 \%)$ had hippocampal metastases (that is $0.97 \%$ of all their metastases). In the group of non-oligometastatic patients, in hippocampus was presented only $2.29 \%$ of BM [59]. Moreover, other study with 371 patients and $1133 \mathrm{BM}$ localize $8.6 \%$ of them into the HA region (hippocampus plus $5 \mathrm{~mm}$ margin); however, no metastasis was presented in the hippocampus itself [60]. It can be concluded, that sparing of hippocampus would likely not significantly increase the risk of treatment failure.

On the other hand, others have hypothesized, that neurogenic niches may not only harbor normal NSC but
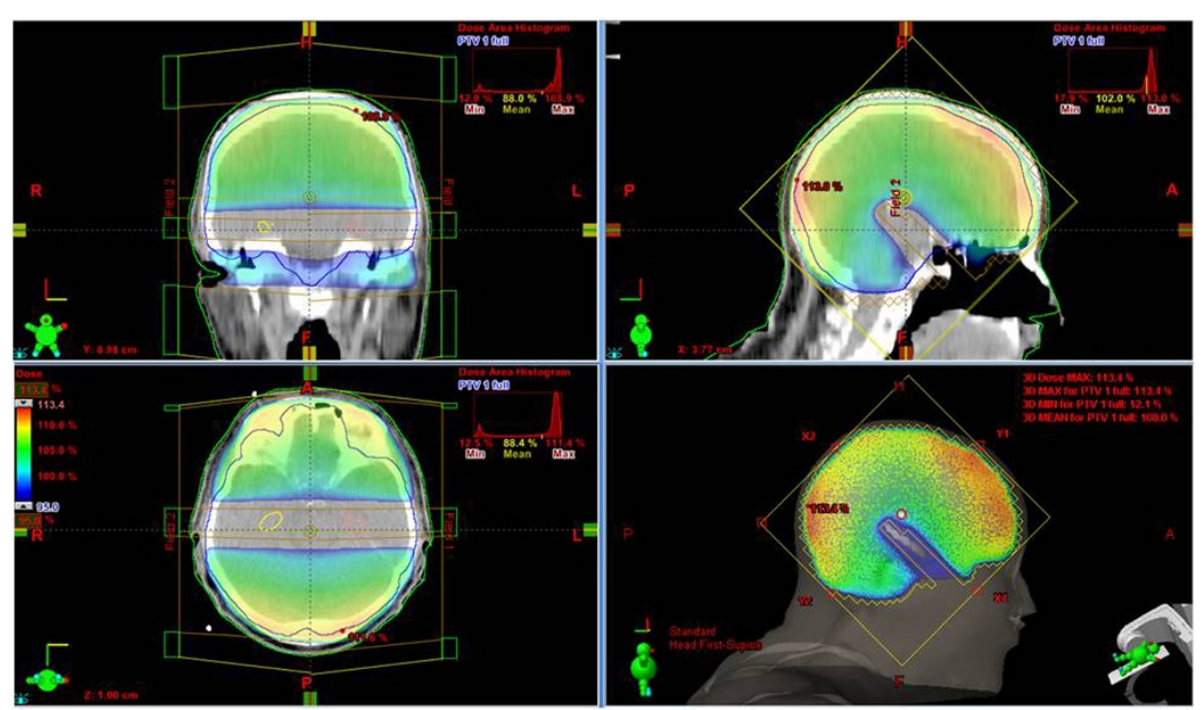

Figure 3 Simple RT technique using 2 laterolateral brain fields with 2 leafs positioned to block the hippocampus. 
also cancer stem cells responsible for late recurrence. In a retrospective analysis of dose coverage of neurogenic niches in patients with malignant gliomas performed by Evers et al., dose to subventricular zone greater than 43 Gy was associated with a significant improvement in progression free survival compared to those with lower dose (15.0 vs. 7.2 months PFS; $\mathrm{P}=0.028$ ) [63]. Interestingly, similar analysis of dose delivered to the hippocampal formation did not yield statistically significant results which confirm the complexity of radiation effects on neurogenic niches [63]. These results highlight the need for well-designed clinical trials as well as continued pre-clinical research to evaluate beneficial or detrimental effects of hippocampal sparing.

The most important treatment related controversy is inconsistency in recommended dose reduction. At this time there is no level I evidence to conclusively support a particular recommendation. Preclinical studies indicate probable no-shoulder dose-response [29]. On the other hand, retrospective clinical studies suggest that biological equivalent dose greater than 7.3 Gy EQD 2 applied to $40 \%$ of hippocampus was associated with worse NCF outcomes [43]. Ongoing phase III trials evaluating NCF function will provide more possible dose-volume constraints associated with possible milder NCF impairment. Because of cost of advanced radiotherapy techniques, it is controversial whether apply intensity modulated plan in some particular patients which will be not able to achieve assumed dose goals especially in situation of standard three dimensional conformal plan would be originally considered. Based on the recently reported phase II trial [11], a dosimetric recommendation for HA WBRT $\left(\mathrm{D}_{\text {median }}<7.8 \mathrm{~Gy}\right.$, $\mathrm{D} 100 \%<10$ Gy and $\left.\mathrm{D}_{\max }<15.3 \mathrm{~Gy}\right)$ can be recommended for patients with brain metastasis and expected survival greater than 6 months.

\section{Future directions}

Although HA appears to be promising in reducing cognitive affects after RT, ongoing studies and other clinical research are needed to determine the optimal dose and volume constraints. It is not clear based on radiobiology of neural stem cell response to radiation whether it is possible to define specific threshold values in terms of recommended target doses. Even if the nature of radiation injury of hippocampus is same in all patients, the different target doses that can reasonably be achieved in different clinical situations vary with prescribed doses and different clinical target volumes i.e. partial vs. whole brain irradiation or in therapeutic vs. prophylactic indication or in adults vs. children brain tumors. The role of $\mathrm{HA}$ in PCI and children has also not been established and is an area of future investigation. Optimal NCF evaluation tools to measure specific effects on hippocampus, as opposed to other etiologies of cognitive dysfunction, and optimal timing of administration is still largely unknown. To be able to compare results from different studies it is necessary to standardize process of NCF testing. However, the ideal tools to measure early changes in cognitive function as compared to late effects of treatment may not be the same. In addition, the testing must be feasible to administer in a busy clinical practice. Ongoing research on pathophysiology of brain irradiation injury may reveal other possible important brain structures whose sparing can contribute to better preservation of NCF, or further analysis of hippocampal subregion (cornu ammonis for example) may demonstrate avoidance region with higher priority for dose sparing. Development of costeffectiveness analysis will be probably one of the most important steps forward to implementation of this advanced radiotherapy technique especially in low and middle income countries. Comparing cost of standard 3D-CRT (classical 2 latero-lateral fields for WBRT for example) and cost of VMAT or helical tomotherapy systems for example poses important questions whether consequent increase in costs offset theoretical mitigation of neurocognitive decline related to brain irradiation. Especially in situation where are presented many other different sources of cognition impairment in patients suffering from advanced cancer.

\section{Conclusion}

In summary, it is now technically and dosimetrically feasible to implement HA approaches into clinical practice. Furthermore, taking into account very low beam-on time of modern RT systems, it is ethically justifiable to use these techniques also in the palliative indications for patients with BM as well as HGG. As regards boosting of BM, comprehensive techniques with SIB provide tumor doses comparable with sequential approach of classical WBRT + SRS which require even 2 planning procedures which can result in such dosimetric inaccuracies. Moreover, HA WBRT with SIB provides better hippocampal sparing and this treatment approach seems to be the most promising to implement into clinical practice after confirmation of better cognitive outcomes after sparing of hippocampus in ongoing clinical trials.

Recently, first phase II clinical trial with prospectively measured NCF while providing HA WBRT showed significantly better outcomes for patients treated with hippocampal sparing in terms of better cognitive functions as well as quality of life. Conventional techniques of WBRT are now still recommended as standard approach for patients with multiple brain metastases and hippocampal sparing is generally not used outside of the context of clinical trials. Phase III studies are now ongoing and further implementation will depend on the results of these trials. For treatment of PBT, especially in its hemispherical location, it is reasonable to include contralateral hippocampus 
into the OARs assuming that no other organ at risks or target volumes would be over/under irradiated. Ongoing phase III trials will definitely prove the clinical significance of this developing approach.

\section{Abbreviations}

BT: Brain tumors; PBT: Primary brain tumors; BM: Brain metastasis; PBRT: Partial brain irradiation; HGG: High-grade gliomas; WBRT: Whole brain radiotherapy; SRS: Stereotactic radiosurgery; AE: Adverse effects; NCF: Neurocognitive functions; PCl: Prophylactic cranial irradiation; HA: Hippocampal avoidance; IMRT: Intensity modulated radiotherapy; NSCs: Neural stem cells; OARs: Organs at risks; LINAC: Linear accelerator; VMAT: Volumetric modulated arc therapy; SIB: Simultaneous integrated boost.

\section{Competing interests}

The authors declare that they have no competing interests.

\section{Authors' contributions}

TK, PP, PS designed the study. TK made the manuscript concept and drafted the article. RJ and NNL provided critical revision and were involved in drafting the manuscript. PB, MS, LH performed the literature search and extracted relevant articles. OS, TP, MV added important content as pictures or tables and included data from their department. All authors participated on revising of the manuscript. All authors read and approved the final manuscript.

\section{Acknowledgements}

Supported by European Regional Development Fund - Project FNUSA-ICRC (No. CZ.1.05/1.1.00/02.0123) and by Support of Study Stays of Czech Researchers Abroad II: Young Talent Incubator (CZ.1.07/2.3.00/20.0117). Supported by MH CZ - DRO (MMCl, 00209805).

Supported by grants IGA NT/14600, NT/14120 of the Czech Ministry of Health.

\section{Author details}

'Department of Radiation Oncology, Faculty of Medicine, Masaryk University and Masaryk Memorial Cancer Institute, Zluty kopec 7, 65653 Brno, Czech Republic. International Clinical Research Center, St. Anne's University Hospital Brno, Pekarska 53, 65691 Brno, Czech Republic. ${ }^{3}$ Department of Neurosurgery, Faculty of Medicine, Masaryk University and St. Anne's University Hospital Brno, Pekarska53, 65691 Brno, Czech Republic ${ }^{4}$ Department of Medical Physics, Masaryk Memorial Cancer Institute, Zluty kopec 7, 65653 Brno, Czech Republic. 'Department of Radiation Oncology, Mayo Clinic, 200 First Street SW, Rochester, MN 55905, USA.

Received: 4 April 2014 Accepted: 7 June 2014

Published: 16 June 2014

\section{References}

1. Siegel R, Ma J, Zou Z, Jemal A: Cancer statistics, 2014. CA Cancer J Clin 2014, 64(1):9-29.

2. Larson DA, Rubenstein JL, McDermott MW: Metastatic Cancer to the Brain. In Devita, Hellman \& Rosenberg's Cancer: Principles \& Practice of Oncology. 8th edition. Edited by DeVita VT, Lawrence TS, Rosenberg SA. Philadelphia: Lippincott Williams \& Wilkins; 2001:2461-2475.

3. Laack NN, Brown PD: Cognitive sequelae of brain radiation in adults. Semin Oncol 2004, 31(5):702-713.

4. Caine C, Mehta MP, Laack NN, Gondi V: Cognitive function testing in adult brain tumor trials: lessons from a comprehensive review. Expert Rev Anticancer Ther 2012, 12(5):655-667.

5. Abe $\mathrm{E}$, Aoyama $\mathrm{H}$ : The role of whole brain radiation therapy for the management of brain metastases in the era of stereotactic radiosurgery. Curr Oncol Rep 2012, 14(1):79-84.

6. Gondi V, Tomé WA, Mehta MP: Why avoid the hippocampus? A comprehensive review. Radiother Oncol 2010, 97(3):370-376.

7. Chen XJ, Xiao JP, Li XP, Jiang XS, Zhang Y, Xu YJ, Dai JR, Li YX: Risk factors of distant brain failure for patients with newly diagnosed brain metastases treated with stereotactic radiotherapy alone. Radiat Oncol 2011, 6:175.

8. Aoyama H, Shirato H, Tago M, Nakagawa K, Toyoda T, Hatano K, Kenjyo M, Oya N, Hirota S, Shioura H, Kunieda E, Inomata T, Hayakawa K, Katoh N, Kobashi G: Stereotactic radiosurgery plus whole-brain radiation therapy vs stereotactic radiosurgery alone for treatment of brain metastases: a randomized controlled trial. JAMA 2006, 295(21):2483-2491.

9. Meyers CA, Brown PD: Role and relevance of neurocognitive assessment in clinical trials of patients with CNS tumors. J Clin Oncol 2006, 24(8):1305-1309.

10. Brown PD, Pugh S, Laack NN, Wefel JS, Khuntia D, Meyers C, Choucair A, Fox S, Suh JH, Roberge D, Kavadi V, Bentzen SM, Mehta MP, Watkins-Bruner D, Radiation Therapy Oncology Group (RTOG): Memantine for the prevention of cognitive dysfunction in patients receiving whole-brain radiotherapy: a randomized, double-blind, placebo-controlled trial. Neuro Oncol 2013, 15(10):1429-1437.

11. Gondi V, Mehta MP, Pugh S, Tome WA, Kanner A, Caine C, Rowley H, Kundapur V, Greenspoon JN, Kachnic L: Memory preservation with conformal avoidance of the hippocampus during whole-brain radiation therapy for patients with brain metastases: primary endpoint results of RTOG 0933. Int J Radiat Oncol Biol Phys 2013, 87(5):1186.

12. Meyers $C A$, Rock EP, Fine HA: Refining endpoints in brain tumor clinical trials. J Neurooncol 2012, 108(2):227-230.

13. Tallet AV, Azria D, Barlesi F, Spano JP, Carpentier AF, Gonçalves A, Metellus $P$ : Neurocognitive function impairment after whole brain radiotherapy for brain metastases: actual assessment. Radiat Oncol 2012, 7:77.

14. Raber J, Rola R, LeFevour A, Morhardt D, Curley J, Mizumatsu S, VandenBerg $S R$, Fike JR: Radiation-induced cognitive impairments are associated with changes in indicators of hippocampal neurogenesis. Radiat Res 2004, 162(1):39-47.

15. Lautin A: Limbic Brain. New York: Kluwer Academic/Plenum; 2001.

16. Tofilon PJ, Fike JR: The radioresponse of the central nervous system: a dynamic process. Radiat Res 2000, 153(4):357-370.

17. Wong CS, Van der Kogel AJ: Mechanisms of radiation injury to the central nervous system: implications for neuroprotection. Mol Interv 2004, 4(5):273-284.

18. Cameron HA, Woolley CS, McEwen BS, Gould E: Differentiation of newly born neurons and glia in the dentate gyrus of the adult rat. Neuroscience 1993, 56(2):337-344.

19. Barani IJ, Benedict SH, Lin PS: Neural stem cells: implications for the conventional radiotherapy of central nervous system malignancies. Int J Radiat Oncol Biol Phys 2007, 68(2):324-333.

20. Gage FH, Kempermann G, Palmer TD, Peterson DA, Ray J: Multipotent progenitor cells in the adult dentate gyrus. J Neurobiol 1998, 36(2):249-266

21. Song $H$, Stevens $C F$, Gage FH: Astroglia induce neurogenesis from adult neural stem cells. Nature 2002, 417(6884):39-44.

22. Palmer TD, Willhoite AR, Gage FH: Vascular niche for adult hippocampal neurogenesis. J Comp Neurol 2000, 425(4):479-494.

23. Bellinzona M, Gobbel GT, Shinohara C, Fike JR: Apoptosis is induced in the subependyma of young adult rats by ionizing irradiation. Neurosci Lett 1996, 208(3):163-166.

24. Peissner $W$, Kocher $M$, Treuer $H$, Gillardon F: lonizing radiation-induced apoptosis of proliferating stem cells in the dentate gyrus of the adult rat hippocampus. Brain Res Mol Brain Res 1999, 71(1):61-68.

25. Yoneoka Y, Satoh M, Akiyama K, Sano K, Fujii Y, Tanaka R: An experimental study of radiation-induced cognitive dysfunction in an adult rat model. Br J Radiol 1999, 72(864):1196-1201.

26. Abayomi OK: Pathogenesis of irradiation-induced cognitive dysfunction. Acta Oncol 1996, 35(6):659-663.

27. Mizumatsu S, Monje ML, Morhardt DR, Rola R, Palmer TD, Fike JR: Extreme sensitivity of adult neurogenesis to low doses of X-irradiation. Cancer Res 2003, 63(14):4021-4027.

28. Bálentová S, Hajtmanová E, Kinclová I, Lehotský J, Dobrota D, Adamkov M: Radiation-induced long-term alterations in hippocampus under experimental conditions. Klin Onkol 2012, 25(2):110-116.

29. Monje ML, Mizumatsu S, Fike JR, Palmer TD: Irradiation induces neural precursor-cell dysfunction. Nat Med 2002, 8(9):955-962.

30. Lee WH, Warrington JP, Sonntag WE, Lee YW: Irradiation alters MMP-2/ TIMP-2 system and collagen type IV degradation in brain. Int I Radiat Oncol Biol Phys 2012, 82(5):1559-1566.

31. Greene-Schloesser D, Robbins ME, Peiffer AM, Shaw EG, Wheeler KT, Chan MD: Radiation-induced brain injury: a review. Front Oncol 2012, 2:73.

32. Fike JR, Rosi S, Limoli CL: Neural precursor cells and central nervous system radiation sensitivity. Semin Radiat Oncol 2009, 19(2):122-132. 
33. Acharya MM, Lan ML, Kan VH, Patel NH, Giedzinski E, Tseng BP, Limoli CL: Consequences of ionizing radiation-induced damage in human neural stem cells. Free Radic Biol Med 2010, 49(12):1846-1855.

34. Limoli CL, Giedzinski E, Rola R, Otsuka S, Palmer TD, Fike JR: Radiation response of neural precursor cells: linking cellular sensitivity to cell cycle checkpoints, apoptosis and oxidative stress. Radiat Res 2004, 161(1):17-27.

35. Parihar VK, Limoli CL: Cranial irradiation compromises neuronal architecture in the hippocampus. Proc Natl Acad Sci U S A 2013, 110(31):12822-12827.

36. Lawrence YR, Li XA, el Naqa I, Hahn CA, Marks LB, Merchant TE, Dicker AP: Radiation dose-volume effects in the brain. Int $J$ Radiat Oncol Biol Phys 2010, 76(Suppl 3):S20-S27.

37. Gondi V, Tolakanahalli R, Mehta MP, Tewatia D, Rowley H, Kuo JS, Khuntia D, Tomé WA: Hippocampal-sparing whole-brain radiotherapy: a "how-to" technique using helical tomotherapy and linear accelerator-based intensity-modulated radiotherapy. Int J Radiat Oncol Biol Phys 2010, 78(4):1244-1252

38. Marsh JC, Godbole R, Diaz AZ, Gielda BT, Turian JV: Sparing of the hippocampus, limbic circuit and neural stem cell compartment during partial brain radiotherapy for glioma: a dosimetric feasibility study. J Med Imaging Radiat Oncol 2011, 55(4):442-449.

39. van Kesteren Z, Belderbos J, van Herk M, Olszewska A, Lamers E, De Ruysscher D, Damen E, van Vliet-Vroegindeweij C: A practical technique to avoid the hippocampus in prophylactic cranial irradiation for lung cancer. Radiother Oncol 2012, 102(2):225-227.

40. Hall EJ, Giacci AJ: Dose-Response Relationships for Model Normal Tissues. In Radiobiology for the Radiologist. 6th edition. Edited by Hall EJ, Giacci AJ. Philadelphia: Lippincott Williams \& Wilkins; 2006:303-326.

41. Merchant TE, Kiehna EN, Kun LE, Mulhern RK, Li C, Xiong X, Boop FA Sanford RA: Phase II trial of conformal radiation therapy for pediatric patients with craniopharyngioma and correlation of surgical factors and radiation dosimetry with change in cognitive function. J Neurosurg 2006, 104(2 Suppl):94-102.

42. Merchant TE, Conklin HM, Wu S, Lustig RH, Xiong X: Late effects of conformal radiation therapy for pediatric patients with low-grade glioma: prospective evaluation of cognitive, endocrine, and hearing deficits. J Clin Oncol 2009, 27(22):3691-3697.

43. Gondi V, Hermann BP, Mehta MP, Tomé WA: Hippocampal dosimetry predicts neurocognitive function impairment after fractionated stereotactic radiotherapy for benign or low-grade adult brain tumors. Int J Radiat Oncol Biol Phys 2012, 83(4):e487-e493.

44. Taylor BF, Picone J, Kimmett J, Chen Z, Knisely JPS: Improved Hippocampal-sparing using an asymmetric conformal Arc technique for treatment of pituitary lesions. Int J Radiat Oncol Biol Phys 2011, 81(2):S291-S292.

45. Marsh JC, Godbole R, Diaz A, Herskovic A, Turian J: Feasibility of cognitive sparing approaches in children with intracranial tumors requiring partial brain radiotherapy: a dosimetric study using tomotherapy. $J$ Cancer Ther Res 2012, 1:1.

46. Nevelsky A, leumwananonthachai N, Kaidar-Person O, Bar-Deroma R, Nasrallah $\mathrm{H}$, Ben-Yosef $\mathrm{R}$, Kuten A: Hippocampal-sparing whole-brain radiotherapy using Elekta equipment. J App/ Clin Med Phys 2013, 14(3):4205.

47. Gutiérrez AN, Westerly DC, Tomé WA, Jaradat HA, Mackie TR, Bentzen SM, Khuntia D, Mehta MP: Whole brain radiotherapy with hippocampal avoidance and simultaneously integrated brain metastases boost: a planning study. Int J Radiat Oncol Biol Phys 2007, 69(2):589-597.

48. Prokic V, Wiedenmann N, Fels F, Schmucker M, Nieder C, Grosu AL: Whole brain irradiation with hippocampal sparing and dose escalation on multiple brain metastases: a planning study on treatment concepts. Int J Radiat Oncol Biol Phys 2013, 85(1):264-270.

49. Awad R, Fogarty G, Hong A, Kelly P, Ng D, Santos D, Haydu L: Hippocampal avoidance with volumetric modulated arc therapy in melanoma brain metastases - the first Australian experience. Radiat Oncol 2013, 8:62-67.

50. Hsu F, Carolan H, Nichol A, Cao F, Nuraney N, Lee R, Gete E, Wong F, Schmuland M, Heran M, Otto K: Whole brain radiotherapy with hippocampal avoidance and simultaneous integrated boost for 1-3 brain metastases: a feasibility study using volumetric modulated arc therapy. Int J Radiat Oncol Biol Phys 2010, 76(5):1480-1485.

51. Marsh JC, Gielda BT, Herskovic AM, Wendt JA, Turian JV: Sparing of the hippocampus and limbic circuit during whole brain radiation therapy: a dosimetric study using helical tomotherapy. J Med Imaging Radiat Oncol 2010, 54(4):375-382.

52. Marsh JC, Godbole RH, Herskovic AM, Gielda BT, Turian JV: Sparing of the neural stem cell compartment during whole-brain radiation therapy: a dosimetric study using helical tomotherapy. Int J Radiat Oncol Biol Phys 2010, 78(3):946-954.

53. Konrad C, Ukas T, Nebel C, Arolt V, Toga AW, Narr KL: Defining the human hippocampus in cerebral magnetic resonance images-an overview of current segmentation protocols. Neuroimage 2009, 47(4):1185-1195.

54. Stěpán-Buksakowska I, Szabó N, Horínek D, Tóth E, Hort J, Warner J, Charvát F, Vécsei L, Roček M, Kincses ZT: Cortical and subcortical atrophy in Alzheimer disease: parallel atrophy of thalamus and hippocampus. Alzheimer Dis Assoc Disord 2014, 28(1):65-72.

55. Hippocampal contouring: a contouring Atlas for RTOG 0933. [http:// www.rtog.org/LinkClick.aspx?fileticket=59vaU8vfgQc\%3d\&tabid=338]

56. Chera BS, Amdur RJ, Patel P, Mendenhall WM: A radiation oncologist's guide to contouring the hippocampus. Am J Clin Oncol 2009, 32(1):20-22.

57. Marsh JC, Ziel GE, Diaz AZ, Wendt JA, Gobole R, Turian JV: Integral dose delivered to normal brain with conventional intensity-modulated radiotherapy (IMRT) and helical tomotherapy IMRT during partial brain radiotherapy for high-grade gliomas with and without selective sparing of the hippocampus, limbic circuit and neural stem cell compartment. $J$ Med Imaging Radiat Oncol 2013, 57(3):378-383.

58. Boehling NS, Grosshans DR, Bluett JB, Palmer MT, Song X, Amos RA, Sahoo N, Meyer JJ, Mahajan A, Woo SY: Dosimetric comparison of three-dimensional conformal proton radiotherapy, intensity-modulated proton therapy, and intensity-modulated radiotherapy for treatment of pediatric craniopharyngiomas. Int J Radiat Oncol Biol Phys 2012, 82(2):643-652.

59. Marsh JC, Herskovic AM, Gielda BT, Hughes FF, Hoeppner T, Turian J, Abrams RA: Intracranial metastatic disease spares the limbic circuit: a review of 697 metastatic lesions in 107 patients. Int J Radiat Oncol Biol Phys 2010, 76(2):504-512.

60. Gondi V, Tome WA, Marsh J, Struck A, Ghia A, Turian JV, Bentzen SM, Kuo JS, Khuntia D, Mehta MP: Estimated risk of perihippocampal disease progression after hippocampal avoidance during whole-brain radiotherapy: safety profile for RTOG 0933. Radiother Oncol 2010, 95(3):327-331.

61. Ghia A, Tomé WA, Thomas S, Cannon G, Khuntia D, Kuo JS, Mehta MP: Distribution of brain metastases in relation to the hippocampus: implications for neurocognitive functional preservation. Int J Radiat Oncol Biol Phys 2007, 68(4):971-977.

62. Hong AM, Suo C, Valenzuela M, Haydu LE, Jacobsen KD, Reisse CH, Fogarty G: Low incidence of melanoma brain metastasis in the hippocampus. Radiother Oncol 2014, 111(1):59-62.

63. Evers P, Lee PP, DeMarco J, Agazaryan N, Sayre JW, Selch M, Pajonk F: Irradiation of the potential cancer stem cell niches in the adult brain improves progression-free survival of patients with malignant glioma. BMC Cancer 2010, 10:384

doi:10.1186/1748-717X-9-139

Cite this article as: Kazda et al:: Why and how to spare the hippocampus during brain radiotherapy: the developing role of hippocampal avoidance in cranial radiotherapy. Radiation Oncology 2014 9:139.

\section{Submit your next manuscript to BioMed Central and take full advantage of:}

- Convenient online submission

- Thorough peer review

- No space constraints or color figure charges

- Immediate publication on acceptance

- Inclusion in PubMed, CAS, Scopus and Google Scholar

- Research which is freely available for redistribution 